CAPÍTULO 6

\title{
Caracterização de óleo essencial extraído de folhas de Citrus bergamia
}

Franciene Almeida Villanova, Dianini Hüttner Kringel, Roberto Pedroso de Oliveira, Caroline Dellinghausen Borges

https://doi.org/10.4322/mp.978-65-991393-3-8.c6

\section{Resumo}

Óleos essenciais podem ser obtidos de plantas e condimentos e destacam-se por suas propriedades antimicrobianas e antioxidantes, o que os tornam interessantes para a utilização na indústria de alimentos, farmacêutica e de embalagens. Objetivouse com o presente estudo caracterizar o óleo essencial (OE) obtido de folhas de Citrus bergamia através do rendimento de extração, da determinação de componentes individuais, da atividade antimicrobiana e da capacidade antioxidante. O OE de Citrus bergamia apresentou rendimento de $0,75 \%$, tendo como componentes majoritários o linalol e o acetato de linalila. Pelos testes CIM e CBM, o OE demonstrou atividade frente à Salmonella Typhimurium, Pseudomonas aeruginosa, Escherichia coli, Staphylococcus aureus e Listeria monocytogenes. Para a atividade antioxidante avaliada pelo método ABTS, o OE apresentou boa habilidade na eliminação dos radicais livres, de acordo com outros estudos sobre óleos essenciais de folhas de Citrus.

Palavras-chave: atividade antimicrobiana, atividade antioxidante, composição química, rendimento de extração.

\section{Introdução}

Os óleos essenciais (OE) são constituídos por misturas complexas de compostos voláteis, que apresentam baixa massa molecular e são insolúveis em água (CALO et al., 2015; DIMA; DIMA, 2015). Esses óleos podem ser obtidos de plantas e condimentos, destacam-se por suas propriedades antimicrobianas e antioxidantes, 0 que os tornam interessantes para a utilização na indústria de alimentos, farmacêutica e de embalagens (ATARÉS; CHIRALT, 2016).

A atividade antimicrobiana e antioxidante dos OE está intimamente ligada à presença de terpenos como limoneno, linalol, carvacrol, timol e cresol (CALO et al., 2015; FANCELLO et al., 2016). Os compostos bioativos que possuem capacidade antimicrobiana e antioxidante podem ser extraídos de diversas espécies vegetais, dentre elas as frutas cítricas, cujo OE encontra-se, especialmente, na casca dos frutos, folhas e flores (JING et al., 2014).

Os citros estão entre as culturas mais produzidas no mundo, e seu OE pode ser extraído por destilação ou prensagem a frio (DIMA; DIMA, 2015; MASSON et al., 2016). O Citrus bergamia, conhecido como bergamota, pertence à família Rutaceae e 
caracteriza-se por ser uma planta endêmica da região de Calábria (Itália), onde seu fruto é utilizado na medicina popular como agente hipocolesterolêmico, além do uso como antisséptico e anti-inflamatório (DI DONNA et al., 2014; SALERNO et al., 2015).

A atividade antimicrobiana de OE extraídos de frutas cítricas, como a bergamota, é atribuída principalmente à presença de compostos como D-limoneno, linalol ou citral (ALOUI et al., 2014). Já a atividade antioxidante está relacionada com a presença de compostos fenólicos e carotenoides, além dos terpenos (LÓPEZ-MUÑOZ; ANTONIOPÉREZ; DÍAZ-REYES, 2015; RUSSO et al., 2016).

Os frutos de Citrus bergamia destinam-se, principalmente, à obtenção de óleo essencial, o qual é comercialmente extraído da casca, conforme já relatado em trabalhos anteriores (FURNERI et al., 2012; LAURO et al., 2015; NAVARRA et al., 2014; RUSSO et al., 2016). Entretanto, são limitados os estudos cujo óleo é extraído das folhas e flores (JING et al., 2014).

Neste contexto, objetivou-se com o presente estudo caracterizar o OE obtido de folhas de Citrus bergamia através do rendimento de extração, da determinação de componentes individuais, da atividade antimicrobiana e da capacidade antioxidante.

\section{Material e Métodos}

\subsection{Material}

Folhas de bergamota (Citrus bergamia, Risso et Poiteau), coletadas em agosto de 2016, no município de Montenegro-RS, Brasil.

\subsection{Métodos}

\subsubsection{Extração do OE}

Amostras de folhas frescas de Citrus bergamia foram trituradas (liquidificador) com água destilada na proporção de, aproximadamente, 1:10 (p/v), e submetidas à hidrodestilação utilizando extrator Clevenger. Após $4 \mathrm{~h}$, o OE foi recolhido, acondicionado em frasco de vidro e armazenado em freezer $\left(-18{ }^{\circ} \mathrm{C}\right)$. Após a obtenção do OE, foi determinado o rendimento de extração utilizando a equação 1:

$$
\text { Rendimento de extração }(\%)=\frac{\text { massa do óleo extraída }(\mathrm{mL})}{\text { biomassa vegetal }(\mathrm{mL})} \times 100
$$

\subsubsection{Determinação de componentes individuais por CG-MS}

A determinação dos componentes individuais do OE de folha de bergamota foi realizada por cromatografia gasosa acoplada à espectrometria de massa (CG-MS), utilizando coluna capilar Rtx-5MS (30 m x 0,25 mm x 0,25 $\mu \mathrm{m}$ ). Em um vial de 1,5 mL foram misturados $20 \mu \mathrm{L}$ do OE de bergamota com $480 \mu \mathrm{L}$ de hexano, para posterior injeção em um GCMS Shimadzu QP2010 Ultra com autoinjetor AOC-20i e biblioteca de espectro de massas NIST 2011. 
Os parâmetros de injeção, cromatografia e espectrometria de massas foram configurados segundo Juliani et al. (2008). Injetou-se $1 \mu \mathrm{L}$ de amostra com temperatura do injetor a $200{ }^{\circ} \mathrm{C}$, no modo splitless. O gás de transporte utilizado foi hélio, sob um fluxo de 1,08 mL. $\mathrm{min}^{-1}$ e velocidade linear como modo de controle de fluxo. A temperatura foi mantida a $60{ }^{\circ} \mathrm{C}$ durante $1 \mathrm{~min}$ e depois aumentou-se gradualmente $5^{\circ} \mathrm{C} \cdot \mathrm{min}^{-1}$ até $180^{\circ} \mathrm{C}$ (mantida por $1 \mathrm{~min}$ ). Na sequência, a temperatura foi ajustada para $200{ }^{\circ} \mathrm{C}$ e os espectros de massas foram registrados no intervalo de 40 a $450 \mathrm{~m} / \mathrm{z}$. Os componentes individuais foram identificados pelo seu espectro de massas correspondentes, obtida por comparação com a biblioteca interna. A composição em percentagem foi obtida a partir da medição da integração das áreas sob os picos.

\subsubsection{Avaliação da atividade antimicrobiana}

Para a avaliação da atividade antimicrobiana, realizaram-se testes de difusão em disco, concentração inibitória mínima e concentração bactericida mínima.

\subsubsection{Teste de difusão em disco}

A determinação do espectro de ação do OE foi realizada através da técnica de difusão em disco (CLSI, 2015). Culturas bacterianas (Listeria monocytogenes ATCC 766, Staphylococcus aureus ATCC 25923, Pseudomonas aeruginosa ATCC 15443, Escherichia coli NTC 12900 e Salmonella typhimurium ATCC 14028) foram suspensas em água peptonada $(0,1 \%)$ obtendo a concentração de $10^{8}$ UFC.g ${ }^{-1}(0,5$ McFarland). O inóculo bacteriano foi semeado com swabs estéreis na superfície de placas com Ágar Mueller-Hinton (MH - Oxoid ${ }^{\circledR}$ ), sobre as quais foram dispostos discos de papel estéreis (Laborclin ${ }^{\circledR}$ ) de $6 \mathrm{~mm}$ de diâmetro. Adicionou-se $10 \mu \mathrm{L}$ do OE em cada disco e, após $1 \mathrm{~h}$ de espera para a absorção, as placas foram incubadas a $37^{\circ} \mathrm{C}$. Após $24 \mathrm{~h}$, verificou-se a existência de halos de inibição, quantificando os existentes com paquímetro digital (King.tools ${ }^{\circledR}$ ). Um controle negativo com os discos adicionados de água destilada estéril foi utilizado, e os resultados foram expressos em $\mathrm{mm}$.

\subsubsection{Concentração inibitória mínima (CIM) e concentração bactericida mínima (CBM)}

A CIM foi determinada através do teste de microdiluição em placa (CLSI, 2015) com placas de 96 poços. O OE foi diluído em caldo Brain Heart Infusion (BHI-Oxoid ${ }^{\circledR}$ ), inoculado com as bactérias (L. monocytogenes, S. aureus, P. aeruginosa, E. coli e $S$. Typhimurium) na concentração de $10^{4}$ UFC. $\mathrm{mL}^{-1}$, e adicionado de $1 \%$ de Tween 80 , obtendo-se concentrações de OE de 166,7 a $0,3 \mu \mathrm{L} \cdot \mathrm{mL}^{-1}$. As placas foram incubadas a $36{ }^{\circ} \mathrm{C}$ por $24 \mathrm{~h}$ e, na sequência, adicionou-se $20 \mu \mathrm{L}$ de cloreto de trifenil tetrazólio a $0,5 \%$ em todas cavidades, sendo após incubadas por mais $15 \mathrm{~min}$. A leitura foi realizada em espectrofotômetro de placas (Robonik ${ }^{\circledR}$ Readwel plate) a $625 \mathrm{~nm}$, considerando como CIM a maior diluição, onde não houve crescimento celular. Como controle negativo de crescimento foi utilizado o caldo BHI com OE $\left(50 \mu \mathrm{L} \cdot \mathrm{mL}^{-1}\right)$ sem a inoculação das bactérias, e como controle positivo de crescimento foi utilizado caldo $\mathrm{BHI}$ inoculado as bactérias sem a adição do OE (OJEDA-SANA et al., 2013).

Para detectar a CBM, foram plaqueadas em Ágar BHI alíquotas de $10 \mu \mathrm{L}$ de cada poço, onde houve inibição no teste da CIM. Foi considerada como CBM a menor concentração onde não houve crescimento neste novo meio após $24 \mathrm{~h}$ a $37^{\circ} \mathrm{C}$. Todos os testes foram realizados em triplicata. 


\subsubsection{Avaliação da atividade antioxidante}

A avaliação da atividade antioxidante do OE foi realizada através de reação colorimétrica com o radical ABTS (3-ethylbenzothiazoline-6-sulfonic acid), realizada por espectrofotometria.

A determinação foi realizada segundo o método descrito por Re et al. (1999). Uma solução-mãe de ABTS $(7 \mathrm{mM})$ e persulfato de potássio $(2,45 \mathrm{mM})$ foi preparada pela mistura destes compostos na proporção de 2:1, sendo esta incubada durante 12-16 h. Após, a absorbância da solução foi ajustada com etanol para 0,7 a $734 \mathrm{~nm}$. Uma alíquota de $10 \mu \mathrm{L}$ de amostras reagiu com $2,9 \mathrm{~mL}$ da solução de trabalho ABTS durante 10 min à temperatura ambiente e, então, a absorbância foi medida a $734 \mathrm{~nm}$. O resultado foi expresso em $\mu \mathrm{mol}$ de equivalentes de trolox por $\mathrm{mL}$ de óleo, e em \% de inibição.

\section{Resultados e Discussão}

\subsection{Rendimento de extração}

A hidrodestilação de folhas frescas de Citrus bergamia resultou em um rendimento de extração de óleo essencial de 0,75 $\pm 0,02 \%$. Apesar de não haver dados reportados na literatura sobre o rendimento de extração de OE de folha de Citrus bergamia, estes podem variar de acordo com a fonte vegetal (espécie e porção vegetal), o método de extração, a época de colheita, o tipo de solo, clima e a umidade relativa do ar (BURT, 2004). Em relação à extração do óleo de folhas de outras espécies de Citrus, como Citrus aurantium (0,30\%), Citrus deliciosa (0,20\%), Citrus sunki $(0,36 \%)$ e Citrus limon $(0,29 \%)$, o valor obtido foi superior (ALMEIDA et al., 2012; SANTOS et al., 2012; CAMPELO et al., 2013). No entanto, outras condições e métodos de extração devem ser avaliados a fim de melhorar o resultado obtido.

\subsection{Determinação de componentes individuais por CG-MS}

A composição química do óleo essencial de folhas de Citrus bergamia está apresentada na Tabela 1. Foram encontrados 13 compostos químicos, sendo o linalol o composto majoritário (42,95\%), seguido do acetato de linalila (23,55\%), a-terpineol $(10,17 \%)$, acetato de geranil $(6,90 \%)$ e 2,6-octadienal 3,7-dimetil-acetato (3,67\%). Os demais compostos somados formam aproximadamente $13 \%$ da composição química deste óleo.

Furneri et al. (2012) ao realizarem a determinação dos componentes do OE extraído da casca de bergamota (Citrus bergamia) por cromatografia gasosa encontraram 59 compostos, dentre os quais destacaram-se o limoneno $(25,64$ a $33,69 \%)$, acetato de linalila (20,13 a $27,05 \%)$, linalol (5,49 a 13,23\%), $\gamma$-terpineno (7,35 a 9,64\%) e $\beta$-pineno $(4,53$ a $6,00 \%)$ como os principais compostos voláteis. Comportamento semelhante foi encontrado por Nabiha et al. (2010) ao analisar o óleo essencial de casca de bergamota (Citrus bergamia), em que foi verificada a presença de 46 compostos, sendo o limoneno o componente majoritário $(59,21 \%)$, seguido do acetato de linalila $(16,83 \%)$ e linalol $(9,51 \%)$. Assim, ao avaliar os compostos presentes no OE extraído das folhas com os OE extraídos das cascas de Citrus bergamia pode-se concluir que há distinção entre os compostos e de concentração destes. $E$ em relação à 
composição do OE de Citrus bergamia (tanto da casca como das folhas) com o de outras espécies cítricas, observa-se que o OE de Citrus bergamia caracteriza-se por apresentar maior concentração relativa dos compostos linalol e acetato de linalila (FURNERI et al., 2012).

De modo geral, a composição química dos OE, sejam da mesma ou de diferentes espécies, pode variar em função da parte da planta utilizada para a extração (flores, partes verdes como hastes e folhas, casca, fruto inteiro, pericarpo ou semente), do local de cultivo, do tipo de solo, do grau de maturação e do método de extração (ANTUNES et al., 2017; CALO et al., 2015).

Tabela 1. Composição química do óleo essencial de folha de Citrus bergamia.

\begin{tabular}{lcc}
\hline \multicolumn{1}{c}{ Composto químico } & Tempo de retenção (min) & Concentração relativa (\%) \\
\hline$\beta$-Pineno & 6,1 & 1,13 \\
$\beta$-Mirceno & 6,3 & 1,96 \\
D-Limoneno & 7,3 & 0,86 \\
trans- $\beta$-Ocimeno & 7,5 & 2,23 \\
Z-Ocimeno & 7,8 & 2,23 \\
2-Carene & 8,9 & 0,53 \\
Linalol & 9,3 & 42,95 \\
$\alpha-T e r p i n e o l$ & 11,8 & 10,17 \\
Acetato de linalila & 13,6 & 23,55 \\
2,6-Octadienol, 3,7- & 16,6 & 3,67 \\
dimetil acetato & 17,1 & 6,90 \\
Acetato de geranil & 18,2 & 2,26 \\
Cariofileno & 20,2 & 1,58 \\
Germacreno B & & \\
\hline
\end{tabular}

\subsection{Atividade antimicrobiana}

Os resultados da atividade antimicrobiana avaliada pelas técnicas de disco difusão, CIM e CBM do OE de Citrus bergamia frente a diferentes bactérias patogênicas estão apresentados na Tabela 2. Quanto ao teste de difusão em disco, o OE ocasionou a inibição de quatro das cinco bactérias testadas (Tabela 2), com halo de até 29,00 mm de diâmetro (Escherichia coli). Assim, com exceção da Listeria monocytogenes, o OE extraído das folhas de Citrus bergamia apresentou forte inibição dos micro-organismos avaliados, de acordo com a classificação de Delfino et al. (2008). De acordo com os autores, halos de até $4 \mathrm{~mm}$ são de fraca inibição ao micro-organismo, halos de 5 a 9 $\mathrm{mm}$ de média inibição e maiores que $10 \mathrm{~mm}$ de forte inibição.

Seguindo este mesmo critério, média inibição foi observada por Gomes et al. (2013) para o OE de folhas de Citrus reticulata Blanco contra Escherichia coli e Salmonella epidermidis, com halos de 7,0 e $8,0 \mathrm{~mm}$, respectivamente. Santos et al. (2016) observaram que OE das cascas de Citrus reticulata $v$. tangerine e Citrus auratium var. 
dulcis apresentam média inibição frente a Sthalylococcus aureus e Escherichia coli, com halos de 4,8 e 5,3 mm (S. aureus) e 5,0 e 8,0 mm (E. coli).

Quanto à concentração inibitória e bacteriostática mínima, não foram observadas diferenças no espectro de ação do OE, o qual apresentou uma CIM de $1,17 \mu \mathrm{L} \cdot \mathrm{mL}^{-1} \mathrm{e}$ CBM de 2,34 $\mu \mathrm{L} \cdot \mathrm{mL}^{-1}$.

De acordo com Aligiannis et al. (2001), CIM de até $500 \mu \mathrm{L}$ de extrato ou óleo essencial por mililitro de diluente são inibidores potentes, entre 600 e $1500 \mu \mathrm{L} \cdot \mathrm{mL}^{-1}$ são inibidores moderados e acima de $1600 \mu \mathrm{L}$. $\mathrm{mL}^{-1}$ são inibidores fracos. Dessa forma, seguindo os critérios de Aligiannis et al. (2001), pode-se considerar que o OE de Citrus bergamia é um inibidor potente para todas as bactérias patogênicas testadas.

Utilizando esta classificação, Santos et al. (2016) demonstraram que OE de cascas de Citrus reticulata $v$. tangerine e Citrus auratium var. dulcis apresentam forte inibição (CIM de $40 \mu \mathrm{L} \cdot \mathrm{mL}^{-1}$ e $160 \mu \mathrm{L} \cdot \mathrm{mL}^{-1}$, respectivamente) frente a $E$. coli e a Salmonella, e CBM de $160 \mu \mathrm{L} \cdot \mathrm{mL}^{-1}$ e $320 \mu \mathrm{L} \cdot \mathrm{mL}^{-1}$, respectivamente.

Geralmente, a maioria dos OE apresentam atividade antimicrobiana superior frente a bactérias Gram positivas, o que se deve às diferenças existentes entre a parede celular destes dois grupos de bactérias. Bactérias Gram negativas possuem uma dupla camada fosfolipídica externa, composta por lipopolissacarídeos, consequentemente pelo caráter hidrofóbico desta estrutura, os compostos apolares dos óleos essenciais encontram dificuldade para penetrar na célula bacteriana. Enquanto nas bactérias Gram positivas 90 a 95\% da parede celular é composta por peptideoglicano, facilitando a penetração de componentes apolares (NAZZARO et al., 2013). No presente estudo, observou-se que o OE não foi eficaz na inibição da bactéria Listeria monocytogenes pelo teste de difusão em disco, entretanto pode-se observar atividade do OE nos demais métodos utilizados, o que demonstra a importância da necessidade de avaliação da atividade antimicrobiana por diferentes métodos.

A atividade inibitória do OE está normalmente relacionada à alta concentração de compostos oxigenados, como o linalol e acetato de linalila, que no presente estudo, representam 66,5\% da composição (FANCELLO et al., 2016). Além disso, a ação antimicrobiana pode ser advinda da capacidade dos compostos em penetrar através das membranas bacterianas para o interior das células, inibindo propriedades funcionais e lipofílicas das mesmas (BAJPAI; BAEK; KANG, 2012). Outros fatores que interferem na ação dos óleos contra micro-organismos são as condições de crescimento e extração, que podem afetar as quantidades dos principais componentes antimicrobianos dos EOs e por consequência, sua eficiência e espectro de ação (CALO et al., 2015).

Tabela 2. Atividade antimicrobiana por disco difusão, CIM e CBM do OE extraído de folhas de Citrus bergamia frente a diferentes bactérias.

\begin{tabular}{|c|c|c|c|c|}
\hline \multirow[b]{2}{*}{ Bactéria } & \multirow[b]{2}{*}{ Cepa } & \multicolumn{3}{|c|}{ Análises } \\
\hline & & $\begin{array}{l}\text { Difusão em } \\
\text { disco (mm) }\end{array}$ & $\begin{array}{c}\mathrm{CIM} \\
\left(\mu \mathrm{L} \cdot \mathrm{mL}^{-1}\right)\end{array}$ & $\begin{array}{c}\mathrm{CBM} \\
\left(\mu \mathrm{L} \cdot \mathrm{mL}^{-1}\right)\end{array}$ \\
\hline
\end{tabular}

Gram negativas:

Salmonella typhimurium

ATCC $14028 \quad 13,67 \pm 3,78 \quad 1,17$ 


$\begin{array}{llccc}\text { Pseudomonas aeruginosa } & \text { ATCC } 15442 & 14,67 \pm 2,30 & 1,17 & 2,34 \\ \text { Escherichia coli } & \text { NTC } 12900 & 29,00 \pm 1,41 & 1,17 & 2,34 \\ \text { Gram positivas: } & & & & \\ \text { Staphylococcus aureus } & \text { ATCC } 25923 & 28,00 \pm 4,24 & 1,17 & 2,34 \\ \text { Listeria monocytogenes } & \text { ATCC } 7644 & - & 1,17 & 2,34\end{array}$

Resultados expressos em média \pm desvio padrão, para o halo de inibição. CIM = Concentração inibitória mínima, CBM = Concentração bactericida mínima

\subsection{Atividade antioxidante do óleo essencial (OE)}

O OE apresentou atividade antioxidante de 1,29 $\pm 0,08 \mu \mathrm{mol}$ Trolox equiv. $\mathrm{mL}^{-1}$ de óleo ou 69,12 $\pm 4,11 \%$ de inibição pelo método ABTS (KIM et al., 2002). A partir do resultado encontrado pode-se inferir que, comparando a outros estudos com OE de folhas de Citrus (FANCELLO et al., 2016; ELLOUZE et al., 2012) o OE estudado apresenta boa habilidade de eliminar radicais livres.

Valores similares foram obtidos por Fancelo et al. (2016) ao avaliar a atividade antioxidante de óleo essencial de Citrus limon var. pompia na concentração de 1,25 mg. $\mathrm{mL}^{-1}$ após 50 min de reação. Urbizu-González et al. (2017) encontraram resultados semelhantes para a capacidade antioxidante de OE de folhas de Turnera diffusa (planta medicinal) cultivada em diferentes regiões do México, a qual variou de 60,85 a 72,30 em \% de inibição, dependendo da região geográfica.

De modo geral, a capacidade antioxidante de compostos vegetais pode variar em função de fatores como as condições ambientais do clima e do solo, por diferenças genotípicas e pelos tratos culturais aplicados durante o cultivo (CHEN et al., 2016).

\section{Conclusão}

O OE de folha de Citrus bergamia apresentou rendimento de extração de $0,75 \%$, tendo como componentes majoritários o linalol e o acetato de linalila. Houve influência do método utilizado na avaliação da atividade antimicrobiana, sendo que pelos testes CIM e CBM, O OE apresentou atividade antimicrobiana frente a Salmonella typhimurium, Pseudomonas aeruginosa, Escherichia coli, Staphylococcus aureus e Listeria monocytogenes. Para a atividade antioxidante avaliada pelo método ABTS, o OE apresentou boa habilidade na eliminação dos radicais livres, conforme outros estudos realizados com óleo essencial de folhas de Citrus. Outros métodos de extração devem ser avaliados a fim de melhorar o rendimento do óleo. O OE apresenta potencial de extração para a aplicação em diferentes áreas como na indústria de alimentos.

\section{Referências}

ALIGIANNIS, N. et al. Composition and antimicrobial activity of the essential oil of two Origanum species. Journal of Agricultural and Food Chemistry, v.40, p.4168-4170, 2001. https://doi.org/10.1021/jf001494m.

ALMEIDA, L. A. DA H. et al. Caracterização química do óleo essencial em folhas de Citrus Aurantium L. E C. Deliciosa Ten. In: CONGRESSO BRASILEIRO DE RECURSOS GENÉTICOS, 2012, Belém. Anais[...]. Belém: SBRG, 2012. p.1-4. 
ALOUI, H. et al. Efficacy of the combined application of chitosan and Locust Bean Gum with different citrus essential oils to control postharvest spoilage caused by Aspergillus flavus in dates. International Journal of Food Microbiology, v.170, p. 21-28, 2014. https://doi.org/10.1016/j.ijfoodmicro.2013.10.017.

ANTUNES, M. D. et al. Antimicrobial electrospun ultrafine fibers from zein containing eucalyptus essential oil/cyclodextrin inclusion complex. International Journal of $\begin{array}{llll}\text { Biological Macromolecules, } & \text { v.104, } & \text { p.874-882, }\end{array}$ https://doi.org/10.1016/j.ijbiomac.2017.06.095.

ATARÉS, L.; CHIRALT, A. Essential oils as additives in biodegradable films and coatings for active food packaging. Trends in Food Science \& Technology, v.48, p.51-62, 2016. https://doi.org/10.1016/j.tifs.2015.12.001.

BAJPAI, V. K.; BAEK, K.-H.; KANG, S. C. Control of Salmonella in foods by using essential oils: a review. Food Research International, v.45, p.722-734, 2012. https://doi.org/10.1016/j.foodres.2011.04.052.

BURT, S. Essential oils: their antibacterial properties and potential applications in foods-a review. International Journal of Food Microbiology, v.94, p.223-253, 2004. https://doi.org/10.1016/j.ijfoodmicro.2004.03.022.

CALO, J. R. et al. Essential oils as antimicrobials in food systems - A review. Food Control, 54, 111-119, 2015. https://doi.org/10.1016/j.foodcont.2014.12.040.

CAMPELO, L.M.L. et al. Constituintes químicos e estudos toxicológicos do óleo essencial extraído das folhas de Citrus limon Burn (Rutaceae). Revista Brasileira de Plantas Medicinais, v.15, p.708-716, 2013. https://doi.org/10.1590/S151605722013000500011.

CHEN, H. et al. Variation in total anthocyanin, phenolic contents, antioxidant enzyme and antioxidant capacity among different mulberry (Morus sp.) cultivars in China. Scientia Horticulturae, $\quad$ v.213, 2016. https://doi.org/10.1016/j.scienta.2016.10.036.

CLSI-Clinical and Laboratory Standards Institute Performance Standards for Antimicrobial Disk Susceptibility Tests; Approved Standard, 11 ${ }^{\text {th }}$ edition, 2015.

DELFINO, T. P. C. Sinergismo entre substâncias antimicrobianas e Lactobacillus acidophilus na inibição de Salmonella enteritidis e Salmonella gallinarum. 2008. 80 f. Tese (Doutorado em Microbiologia Agropecuária) - Faculdade de Ciências Agrárias e Veterinárias, Universidade Estadual Paulista Julio De Mesquita Filho, Jaboticabal, 2008.

DI DONNA, L. et al. Hypocholesterolaemic activity of 3-hydroxy-3-methyl-glutaryl flavanones enriched fraction from bergamot fruit (Citrus bergamia): "In vivo" studies. Journal of Functional Foods, v.7, p.558-568, 2014. https://doi.org/10.1016/j.jff.2013.12.029.

DIMA, C.; DIMA, S. Essential oils in foods: extraction, stabilization, and toxicity.

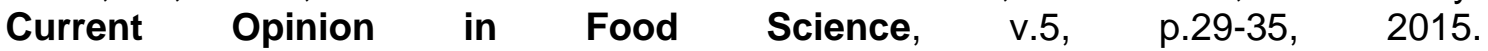
https://doi.org/10.1016/j.cofs.2015.07.003.

ELLOUZE, I. et al. Season's variation impact on Citrus aurantium leaves essential oil: Chemical composition and biological activities. Journal of Food Science, v.77, p.173180, 2012. https://doi.org/10.1111/j.1750-3841.2012.02846.x.

FANCELLO, F. et al. Chemical characterization, antioxidant capacity and antimicrobial activity against food related microorganisms of Citrus limon var. pompia leaf essential 
oil. LWT - Food Science and Technology, v.69, p.579-585, 2016. https://doi.org/10.1016/j.Iwt.2016.02.018.

FURNERI, P. M. et al. In vitro antimycoplasmal activity of citrus bergamia essential oil and its major components. European Journal of Medicinal Chemistry, v.52, p.66-69, 2012. https://doi.org/10.1016/j.ejmech.2012.03.005.

GOMES, F. J. S. et al. Atividade antimicrobiana do óleo essencial de Citrus reticulata Blanco (tangerina) frente à Staphylococcus epidermides e Escherichia coli. In: VII SBOE - SIMPÓSIO BRASILEIRO DE ÓLEOS ESSENCIAIS, 2013, Santarém. Anais [...]. Santarém: UFOPA, 2013. 1p.

JING, L. et al. Antifungal activity of citrus essential oils. Journal of Agricultural and Food Chemistry, v.62, p.3011-3033, 2014. https://doi.org/10.1021/jf5006148.

JULIANI, H. R. et al. Chemical diversity of Lippia multiflora essential oils from West Africa. Journal of Essential Oil Research, v.20, p.49-55, 2008. https://doi.org/10.1080/10412905.2008.9699420.

$\mathrm{KIM}$, D.-O. et al. Vitamin C equivalent antioxidant capacity (VCEAC) of phenolic phytochemicals. Journal of Agricultural and Food Chemistry, v.50, p.3713-3717, 2002. https://doi.org/10.1021/jf020071c.

LAURO, F. et al. The protective role of bergamot polyphenolic fraction on several animal models of pain. PharmaNutrition, v.4, p.S35-S40, 2015. https://doi.org/10.1016/j.phanu.2016.04.001.

LÓPEZ-MUÑOZ, G. A.; ANTONIO-PÉREZ, A.; DÍAZ-REYES, J. Quantification of total pigments in citrus essential oils by thermal wave resonant cavity photopyroelectric $\begin{array}{lllll}\text { spectroscopy. Food Chemistry, v.174, p.104-109, } 2015 . & \end{array}$ https://doi.org/10.1016/j.foodchem.2014.11.039.

MASSON, J. et al. Oxygenated heterocyclic compounds to differentiate Citrus spp. essential oils through metabolomic strategies. Food Chemistry, v.206, p.223-233, 2016. https://doi.org/10.1016/j.foodchem.2016.03.057.

NABIHA, B. et al. Chemical composition of bergamot (Citrus bergamia Risso) essential oil obtained by hydrodistillation. Journal of Chemistry and Chemical Engineering, v.4, p.60-62, 2010.

NAVARRA, M. et al. Effect of Citrus bergamia juice on human neuroblastoma cells in vitro and in metastatic xenograft models. Fitoterapia, v.95, p.83-92, 2014. https://doi.org/10.1016/j.fitote.2014.02.009.

NAZZARO, F. Effect of essential oils on pathogenic bacteria. Pharmaceuticals, v.6, p.1451-1474, 2013. https://doi.org/10.3390/ph6121451.

OJEDA-SANA, A. M. et al. New insights into antibacterial and antioxidant activities of rosemary essential oils and their main components. Food Control, 31, 189-195, 2013. https://doi.org/10.1016/j.foodcont.2012.09.022.

RE, R. et al. Antioxidant activity applying an improved ABTS radical cation decolorization assay. Free Radical Biology \& Medicine, v.26, p.1231-1237, 1999. https://doi.org/10.1016/S0891-5849(98)00315-3.

RUSSO, M. et al. Bergamot (Citrus bergamia Risso) as a source of nutraceuticals: Limonoids and flavonoids. Journal of Functional Foods, v.20, p.10-19, 2016. https://doi.org/10.1016/j.jff.2015.10.005. 
SALERNO, R. et al. Characterization of flavonoids in Citrus bergamia (Bergamot) polyphenolic fraction by liquid chromatography-high resolution mass spectrometry $\begin{array}{lllll}\text { (LC/HRMS). } & \text { PharmaNutrition, } & \text { v.4, } & \text { p. } & \text { S1-S7, }\end{array}$ https://doi.org/10.1016/j.phanu.2015.10.001.

SANTOS, J. Z. DOS et al. Caracterização química dos óleos essenciais em folhas de Citrus sunki hort. Ex. Tanaka e híbrido. In: CONGRESSO BRASILEIRO DE RECURSOS GENÉTICOS, 2012, Belém. Anais[...]. Belém: SBRG, 2012. p.1-4.

SANTOS, A. O. et al. Atividade antibacteriana e antioxidante de óleos essenciais cítricos com potencialidade para inclusão como aditivos em alimentos. Caderno de Ciências Agrárias, v.8, p.15-21, 2016.

URBIZU-GONZÁLEZ, A. L. et al. Natural variability of essential oil and antioxidants in the medicinal plant Turnera diffusa. Asian Pacific Journal of Tropical Medicine, v.10, p.121-125, 2017. https://doi.org/10.1016/j.apjtm.2017.01.013.

\section{Autores}

Franciene Almeida Villanova ${ }^{1}$, Dianini Hüttner Kringel $^{2}$, Roberto Pedroso de Oliveira ${ }^{3}$, Caroline Dellinghausen Borges ${ }^{4, *}$

1. Curso de Pós-Graduação Lato Sensu em Ciência dos Alimentos, Centro de Ciências Químicas, Farmacêuticas e de Alimentos, Universidade Federal de Pelotas, Campus Capão do Leão, Caixa Postal 354, CEP. 96010-900, Pelotas, RS, Brasil

2. Departamento de Ciência e Tecnologia Agroindustrial, Universidade Federal de Pelotas, Campus Capão do Leão, 96010-900, Pelotas, RS, Brasil.

3. Empresa Brasileira de Pesquisa Agropecuária, EMBRAPA, 96010-971, Pelotas, RS, Brasil.

4. Centro de Ciências Químicas, Farmacêuticas e de Alimentos, Universidade Federal de Pelotas, Campus Capão do Leão, Caixa Postal 354, CEP. 96010-900, Pelotas, RS, Brasil

* Autor para correspondência: francienevillanova@hotmail.com 